\title{
Inclusion of Industry Professional Experts in biomedical engineering design courses at-scale
}

\section{Collin W Shale, Johns Hopkins University}

Collin Shale is a junior lecturer with the Department of Biomedical Engineering at Johns Hopkins University. Collin received his bachelor's degree in biomedical engineering from Marquette University, and he received his master's degree in bioengineering innovation and design from Johns Hopkins University, where he worked on projects relating to infection prevention for intravenous infusion and tuberculosis diagnostics. Collin is an instructor for the capstone undergraduate Design Team program, a suite of courses that train student leaders and teams in the design process. In his role, Collin provides instruction on clinical observation, need identification and validation, and opportunity assessment in addition to providing curricular and advisory support to student design teams.

\section{Miss Shababa Binte Matin, Johns Hopkins University}

Shababa Matin has received her combined Bachelor of Arts and Bachelor of Science degree in Visual Arts and Biomedical Engineering from Brown University, followed by a Masters of Science and Engineering from the Center of Bioengineering Innovation and Design at Johns Hopkins University. Since 2019, she has been a junior lecturer at the BME Undergraduate Design Team Program at Johns Hopkins University. She is interested in design as it applies to developing and bringing new healthcare innovations to public and global health spheres.

\section{Mr. Nicholas J Durr, Johns Hopkins University}

Nicholas J. Durr is an Assistant Professor of Biomedical Engineering at Johns Hopkins University and the co-Director of Undergraduate Programs at the Center for Bioengineering Innovation and Design. He leads the Computational Biophotonics Laboratory at Hopkins. He received a B.S. in Electrical Engineering and Computer Science from U.C. Berkeley and a Ph.D. in Biomedical Engineering from U.T. Austin. He was a Postdoctoral Fellow at Harvard Medical School in 2010 and an independent investigator at MIT from 2011 to 2014 as a Fellow in the M+Vision Consortium. Before joining Johns Hopkins, he was the Founder and CEO of PlenOptika.

\section{Elizabeth A Logsdon, The Johns Hopkins University}

Dr. Logsdon is a senior lecturer at Johns Hopkins University in the Department of Biomedical Engineering (BME). She is also the Associated Director of Academic Programs and Director of the BME Design Studio - a facility that supports design efforts in many bioengineering courses at the University and within the Center for Bioengineering Innovation and Design. 


\title{
Work in Progress: Inclusion of Industry Professional Experts in Biomedical Engineering Design Courses at scale
}

\begin{abstract}
Project-based design courses require teaching in diverse subject matters including design, intellectual property (IP), regulation, and market access. For large courses ( $>50$ students) this is often managed by bringing in working professionals as lecture-platform guest speakers. Although the lecture format allows the speaker to reach a larger audience, it fails to provide project-specific information that is critical to decision making in these areas. Additionally, it is challenging to engage a large number of experts to work individually with student teams. A series of expert office hours were derived from the incubator model of mentors-in-residence to support a large two-semester biomedical engineering (BME) design course in three domains: (1) intellectual property (2) regulation (3) market access. These office hours were hosted by IP lawyers, regulatory consultants, and market access professionals. This model was successfully implemented to meet the demand of 110 students (14 teams) for project-specific feedback using a limited number of experts (1-2 per domain) and was widely accepted by the students, with $>95 \%$ of students reporting the model met their needs.
\end{abstract}

Background: The movement towards project-based learning courses has brought new challenges as education transitions from lecture-based delivery to project-specific mentoring. Three commonly addressed domains in BME capstone design courses are IP, regulation, and market access $[1,2,3]$. While guest lectures from industry experts are often used to discuss these domains at-scale, these lectures are often too generalized or not-timed with the project need. Further, frontloading guidance or providing it on predetermined schedules can be ineffective for student utilization. Providing access to domain experts beyond a platform lecture leads to large demands on experts' time. Current methods such as the use of advisory committees for domain expertise, however useful for providing project-specific information, require extensive time commitments from experts. MedTech, biotech and other start-up accelerators and incubators addressed this challenge by using a mentor-in residence model to provide just-in-time guidance [4]. This approach was used as inspiration to develop a more scalable model to provide teams with project-specific feedback from domain experts.

Methods: A series of expert office hours was initiated in a two-semester BME capstone design course to provide a scalable model for project-specific feedback in three domains: intellectual property, regulation, and market access. These office hours were ungraded and not required so teams could opt-in as needed. In year 1 (Y1) office hours provided project-specific guidance in two areas: (1) IP assessment and management and (2) regulation of medical devices and navigating the FDA. A third area, (3) developing a market access strategy, was added to the series in year 2 (Y2). Office hours were hosted by IP lawyers, regulatory consultants, and market access consultants, all of whom previously volunteered their time as guest lecturers or advisory committee members. The primary incentive for their participation was the increased time efficiency offered by this series compared to their current commitments. Secondary interest included desired involvement in education and talent recruitment. During this time period, the course had an average class size of $\sim 110$ students across $\sim 14$ teams. The course deliverables followed a spiral model [5] to encourage students to develop their design projects in increasing depth in clinical, commercial, technical, and strategic areas. Course faculty and clinically derived 
project mentors provided general instruction in all domains during the fall semester, and expert office hours were scheduled in the middle of the spring to provide team-specific feedback on IP, regulation, and market access. Each expert's availability was condensed to one or two blocks which were equally divided into individual team sessions based on team demand. To make efficient and effective use of expert office hours, teams opting-in for an individual session submitted a $<1$-page brief containing domain-relevant background on the project and questions. The experts had a week to prepare for their office hour sessions based on these briefs and used their annotated briefs to guide targeted discussions with each team. Data were collected on the number of teams served by office hours and exit surveys were used to assess student satisfaction and demand for them.

Results: Expert office hours allowed us to provide project-specific feedback to $\sim 14$ different projects each year (Figure 1). Each domain utilized between 1-2 domain experts. Individual team sessions ranged from 12-30 minutes per team. Exit surveys showed that $95 \%$ of students reported that the office hours met their needs. All three expert domains scored similarly (Figure 2). Qualitative feedback from experts was also positive. Expert retention rates for the market access, IP, and regulation domains were 100\% (2/2), 50\% (1/2), and 100\% (1/1), respectively.

\begin{tabular}{|c|c|c|c|c|c|c|c|}
\hline \multirow{2}{*}{ Year } & \multirow{2}{*}{$\begin{array}{c}\text { Total } \\
\text { Teams }\end{array}$} & $\begin{array}{c}\text { Market Access } \\
\text { Total } \\
\text { Hours }\end{array}$ & $\begin{array}{c}\text { \# Teams } \\
\text { Served }\end{array}$ & $\begin{array}{c}\text { Total } \\
\text { Hours }\end{array}$ & $\begin{array}{c}\text { \# Teams } \\
\text { Served }\end{array}$ & $\begin{array}{c}\text { IPotal } \\
\text { Hours }\end{array}$ & $\begin{array}{c}\text { \# Teams } \\
\text { Served }\end{array}$ \\
\hline Y1 & 14 & $\mathrm{NA}^{1}$ & $\mathrm{NA}^{1}$ & 4.5 & 8 & 1.25 & 5 \\
\hline Y2 & 13 & 2 & 10 & 4 & 13 & 2 & $\mathrm{NA}^{2}$ \\
\hline Y3 & 14 & 8 & 12 & 2 & 9 & 2 & 8 \\
\hline
\end{tabular}

Figure 1: Teams served through office hours by expert type. Primary outcomes included total hours committed by experts and the number of teams served. ${ }^{1}$ Market Access office hours were not added until Y2. ${ }^{2}$ In Y2, teams were not required to opt-in or submit a brief for regulatory office hours, and instead showed up to an open working session with the expert and course faculty. This resulted in increased utilization, but the number of teams that interacted solely with the expert was not tracked. We returned to an op-in model in Y3 to improve traceability.

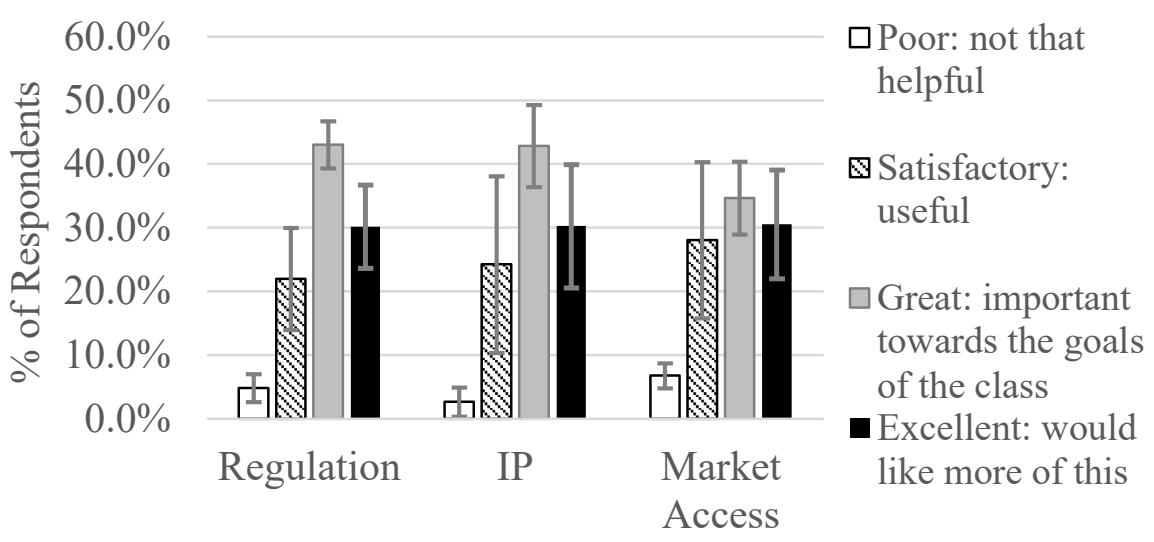

Figure 2: Student exit survey evaluations of expert office hours, by expert type. The percentage frequencies were averaged over Y2 and Y3. No statistics were performed on this data. 
Discussion: Results indicate that the expert office hours model was a scalable way to achieve effective and efficient project-specific guidance in the domains of IP, regulation and market access. The positive impact of personalized feedback from industry experts was qualitatively reflected in the quality of domain strategy discussion during team check-ins. Intimate team meetings with domain experts also resulted in continued deeper relationships with experts that were maintained after the course ended. The opt-in nature of this model allowed teams that were ready access to just-in-time guidance during office hours. Opt-in rates varied by domain, with roughly $50 \%$ of teams using them as scheduled. Additional office hour sessions could be scheduled at later times to service teams that were not ready to opt-in. Since current course deliverables do not assess team progress in the IP and market access domains, the faculty are monitoring the extracurricular success of teams (patenting, business plan competitions, external funding, and co-curricular project continuation) for further impact over the next 5 years.

Conclusion: The implementation of expert office hours successfully administered projectspecific feedback in highly specialized topics at scale ( $\sim 14$ teams), with positive responses from both students and experts. The faculty are exploring further changes, such as adding contract manufacturing experts to provide additional prototyping guidance and implementing a retainer to increase expert availability. Additional project outcomes relating to expert guidance in these domains will continue to be measured for impact over the next 5 years.

\section{References:}

[1] J. Goldberg. "Maintaining a Relevant, Up-to-Date Capstone Design Course [Senior Design]". IEEE Pulse, Vol. 3, No. 1, pp.64-72, Jan. 2012. [Online]. https://doi.org/10.1109/MPUL.2011.2177191. [Accessed Jan 15, 2020].

[2] A. Manbachi, E.A. Logsdon, Y. Yazdi, et al. "Curricular Advancement of Biomedical Engineering Undergraduate Design Projects Beyond 1 Year: A Pilot Study”. Ann Biomed Eng, Vol. 47, No. 12, Dec. 2019 [Online]. Available: https://doi.org/10.1007/s10439-01902434-7. [Accessed Jan. 15, 2020].

[3] A.W. Eberhardt, O.L. Johnson, et al. "Team-Based Development of Medical Devices: An Engineering-Business Collaborative”. J Biomech Eng. Vol. 138, No. 7, Jul. 2016. [Online]. https://doi.org10.1115/1.4032805 [Accessed Jan. 31, 2020]

[4] C. Cohen., D.C. Fehder, et al. "The design of startup accelerators", Research Policy, Vol. 48, No. 7, pp. 1781-1791, Sep. 2019 [Online]. https://doi.org/10.1016/j.respol.2019.04.003

[Accessed Feb. 3, 2020].

[5] Y. Yazdi, S. Acharya. " A New Model for Graduate Education and Innovation in Medical Technology". Ann Biomed Eng, Vol. 41, 1822-1833 (2013). [Online]. https://doi.org/10.1007/s10439-013-0869-4. [Accessed Feb. 3, 2020] 